\title{
Optimal Control of Spacecraft Orbital Maneuvers by the Hamilton-Jacobi Theory
}

\author{
Chandeok Park*, Daniel J. Scheeres ${ }^{\dagger}$
}

\begin{abstract}
Seeking the optimal control of spacecraft orbital maneuvers with non-smooth control logic in feedback sense, we extend our recently developed technique based on the Hamilton-Jacobi theory. Specifically we propose a new methodology stemming from the direct use of generating functions for solving optimal feedback control problem. Starting from the Hamilton-Jacobi equation for generating functions representing a two point boundary value problem, we derive a set of 1st order quasilinear partial differential equations with the associated initial condition, which forms the well-known Cauchy problem. These equations can also be derived by applying the invariant imbedding method to the two point boundary value problem. The solution to this Cauchy problem is utilized for determining the optimal control logic of spacecraft orbital maneuvers with hard and soft constraint boundary conditions. Illustrative examples demonstrate that this approach is, in contrast to the direct use of generating functions, promising for solving problems with non-smooth control logic usually caused by imposing control constraints.
\end{abstract}

\section{Introduction}

We present a novel approach to developing optimal trajectories of spacecraft orbital maneuvers, focusing on problems with non-smooth control logic usually evoked by imposing constraints on control variables. Extending our recently developed technique based on the Hamilton-Jacobi theory, ${ }^{1,2}$ we derive a set of new governing equations for solving the non-smooth optimal control problem in feedback sense. Also this approach does not require us to guess the initial costate (without any physical interpretations) to solve the associated two point boundary value problem.

Since Lawden ${ }^{3}$ initially introduced primer vector theory, optimal control problems for orbital maneuvers using continuous thrust have been a topic of continual interest. While some early works still remain meaningful and noteworthy, ${ }^{4-7}$ many significant results have appeared since $1980 \mathrm{~s} \cdot{ }^{8-13}$ However, it still remains challenging to evaluate the actual optimal trajectories for a variety of orbital maneuvers even in open-loop sense; it requires us to solve the two point boundary value problem (TPBVP) for a Hamiltonian system numerically, which again forces us to guess the initial costate without any physical interpretations. What is worse, the solution to the TPBVP tends to be extremely sensitive to this initial guess in many realistic formulations with bounded control force.

Recently we have studied optimal feedback control (OFC) problems in the context of Hamiltonian systems. Treating the Hamiltonian system derived from the necessary conditions for optimality as a canonical transformation, we have used its generating functions to solve the OFC problem successfully for a class of analytic problems. However, despite many favorable properties, our method has shown restrictive applicability to problems with control constraints mainly due to the difficulty of treating the inherent switching structure and non-smoothness of control logic. ${ }^{14}$

In an attempt to overcome or mitigate these barriers, we present a new technique stemming from the generating function method, and study optimal spacecraft orbital maneuvers extensively with different formulations. The whole discussion is structured as follows. In section II, we formulate the optimal control problem as a Hamiltonian system and briefly review our generating function method as a pre-requisite. In section III, from the Hamilton-Jacobi equations

${ }^{*} \mathrm{PhD}$. Candidate \& Research Assistant, Department of Aerospace Engineering, FXB 1320 Beal Avenue, Ann Arbor, MI 48109-2140, student member AIAA.

${ }^{\dagger}$ Associate Professor, Department of Aerospace Engineering, Associate Fellow AIAA 
for generating functions we derive a set of new governing equations with the appropriate boundary conditions, which forms a Cauchy problem for the Hamiltonian system. We discuss how to obtain the OFC law from its solution, which is followed by some illustrative examples. In section IV, we apply this technique to two different formulations of the non-smooth spacecraft orbital maneuver problems, which shows its own validity and effectiveness through specific numerical examples. Finally concluding remarks follow in section V.

\section{General Optimal Control Problem Nested in the Hamilton-Jacobi Theory}

We consider minimization of the following performance index

$$
J=\phi\left(x\left(t_{f}\right), t_{f}\right)+\int_{t}^{t_{f}} L(x(\tau), u(\tau), \tau) d \tau
$$

subject to the following system with terminal boundary conditions

$$
\dot{x}=F(x, u, t), \psi\left(x\left(t_{f}\right), t_{f}\right)=0
$$

Here $x \in \mathbf{R}^{n}, u \in \mathbf{R}^{m}, t \in \mathbf{R}, \phi\left(x\left(t_{f}\right), t_{f}\right): \mathbf{R}^{n} \times \mathbf{R} \rightarrow \mathbf{R}, L(x(\tau), u(\tau), \tau): \mathbf{R}^{n} \times \mathbf{R}^{m} \times \mathbf{R} \rightarrow \mathbf{R}, F(x(t), u(t), t):$ $\mathbf{R}^{n} \times \mathbf{R}^{m} \times \mathbf{R} \rightarrow \mathbf{R}^{n}$, and $\psi\left(x\left(t_{f}\right), t_{f}\right): \mathbf{R}^{n} \times \mathbf{R} \rightarrow \mathbf{R}^{p \leq n}$. The control $u=\left[\begin{array}{lll}u_{1} & u_{2} \cdots u_{m}\end{array}\right]^{T}$ is bounded by the following inequality by component:

$$
\left|u_{i}\right| \leq u_{i 0}=\text { constant }
$$

The unconstrained problem can be dealt with by letting $u_{i 0} \rightarrow \infty, i=1,2, \cdots, m$.

Given this problem statement, we desire to find the optimal control logic in feedback sense for a given domain in $(x, t) \in \mathbf{R}^{n} \times \mathbf{R}$. Then from any initial point, we can evaluate the optimal trajectory satisfying the terminal constraints by simple forward integration of the system (1), updating the control as new state measurements are made.

Instead of resorting to dynamic programming and solving the Hamilton-Jacobi-Bellman equation (HJBE), we formulate the given problem as a Hamiltonian system. First define the pre-Hamiltonian $\bar{H}$ as

$$
\bar{H}(x, \lambda, u, t)=L(x, u, t)+\lambda^{T} F(x, u, t) .
$$

Then, Pontryagin's principle provides the necessary conditions for optimality and defines a Hamiltonian system for states and costates only ${ }^{1,15}$ :

$$
\begin{aligned}
H(x, \lambda, t) & =\bar{H}\left(x, \lambda, u^{*}(x, \lambda, t), t\right) \\
\dot{x} & =H_{\lambda}(x, \lambda, t) \\
\dot{\lambda} & =-\frac{\partial H}{\partial x}(x, \lambda, t) \\
u^{*}(x, \lambda, t) & =\arg \min _{\bar{u}} \bar{H}(x, \lambda, \bar{u}, t)
\end{aligned}
$$

As is noted in the problem definition, the initial states are chosen explicitly on a given domain. For the terminal condition, suppose we have an explicit condition for $\psi$ :

$$
\psi\left(x\left(t_{f}\right), t_{f}\right)=x\left(t_{f}\right)-x_{f}=0
$$

where $x_{f} \in \mathbf{R}^{n}$ is a constant vector. Then the terminal states are completely specified, which forms the hard constraint problem. Otherwise if $\psi\left(x\left(t_{f}\right), t_{f}\right)=0$ is given by an implicit equation or does not exist, then the following transversality condition determines the $n$ terminal boundary conditions [15, section 2$]$ :

$$
\begin{gathered}
\lambda\left(t_{f}\right)=\frac{\partial\left[\phi\left(x\left(t_{f}\right), t_{f}\right)+\nu^{T} \psi\left(x\left(t_{f}\right), t_{f}\right)\right]}{\partial x\left(t_{f}\right)} \\
2 \text { of } 16
\end{gathered}
$$


In the sense that the terminal states are not directly specified but indirectly affected by $\phi$ and $\psi$, we call this type of boundary condition the soft constraint problem. In either case we have $2 n$ split boundary conditions equally divided between the initial and terminal time. Therefore, the optimal control problem is reduced to a two point boundary value problem (TPBVP).

There exists diverse numerical techniques for solving this TPBVP, which usually yield the open loop optimal trajectory. However, this does not fit into our purpose of obtaining a feedback control scheme on a given domain. Instead, we view the Hamiltonian phase flow $(x(t), \lambda(t))$ as a transformation between terminal coordinates $(x, \lambda, t)$ and initial coordinates $\left(x_{0}, \lambda_{0}, t_{0}\right)$, which is by definition a canonical transformation. Then there exist generating functions for these transformations that can have one of the four classical forms: ${ }^{16-18}$

$$
F_{1}\left(x, x_{0}, t, t_{0}\right), F_{2}\left(x, \lambda_{0}, t, t_{0}\right) F_{3}\left(\lambda, x_{0}, t, t_{0}\right), F_{4}\left(\lambda, \lambda_{0}, t, t_{0}\right)
$$

Note that these generating functions are functions of $n$ initial coordinates and $n$ terminal coordinates. By definition they satisfy the given boundary value problem and provide relations between initial and terminal states and costates by the following relations ${ }^{17}$ :

$$
\begin{aligned}
& \lambda=\frac{\partial F_{1}\left(x, x_{0}, t, t_{0}\right)}{\partial x} \\
& \lambda_{0}=-\frac{\partial F_{1}\left(x, x_{0}, t, t_{0}\right)}{\partial x_{0}} \\
& 0=H(x, \lambda, t)+\frac{\partial F_{1}\left(x, x_{0}, t, t_{0}\right)}{\partial t} \\
& \lambda=\frac{\partial F_{2}\left(x, \lambda_{0}, t, t_{0}\right)}{\partial x} \\
& x_{0}=\frac{\partial F_{2}\left(x, \lambda_{0}, t, t_{0}\right)}{\partial \lambda_{0}} \\
& 0=H(x, \lambda, t)+\frac{\partial F_{2}\left(x, \lambda_{0}, t, t_{0}\right)}{\partial t} \\
& x=-\frac{\partial F_{3}\left(\lambda, x_{0}, t, t_{0}\right)}{\partial \lambda} \\
& \lambda_{0}=-\frac{\partial F_{3}\left(\lambda, x_{0}, t, t_{0}\right)}{\partial x_{0}} \\
& 0=H(x, \lambda, t)+\frac{\partial F_{3}\left(\lambda, x_{0}, t, t_{0}\right)}{\partial t} \\
& x=\frac{\partial F_{4}\left(\lambda, \lambda_{0}, t, t_{0}\right)}{\partial \lambda} \\
& x_{0}=-\frac{\partial F_{4}\left(\lambda, \lambda_{0}, t, t_{0}\right)}{\partial \lambda_{0}} \\
& 0=H(x, \lambda, t)+\frac{\partial F_{4}\left(\lambda, \lambda_{0}, t, t_{0}\right)}{\partial t} .
\end{aligned}
$$

As can be seen, the generating functions satisfy a partial differential equation found by substituting for $\lambda$ in (11) and (14), and for $x$ in (17) and (20), which are usually referred to as the Hamilton-Jacobi equation (HJE).

A crucial property of the generating functions related to a given transformation is that they are linked to each other 
via Legendre transformations, which can be represented by the following identities:

$$
\begin{aligned}
& F_{2}\left(x, \lambda_{0}, t, t_{0}\right)=F_{1}\left(x, x_{0}, t, t_{0}\right)+\lambda_{0}^{T} x_{0} \\
& F_{3}\left(\lambda, x_{0}, t, t_{0}\right)=F_{1}\left(x, x_{0}, t, t_{0}\right)-\lambda^{T} x \\
& F_{4}\left(\lambda, \lambda_{0}, t, t_{0}\right)=F_{2}\left(x, \lambda_{0}, t, t_{0}\right)-\lambda^{T} x
\end{aligned}
$$

Among these generating functions, $F_{1}$ is a special quantity for the optimal control problem as it provides the optimal cost function by the following theorem:

Theorem II.1 (Optimal Cost and Control Law from $F_{1}$ ) Let $x_{f}$ be the (fixed) terminal state at $t_{f}$ and $x$ be the (moving) initial state at $t$. Also let $F_{1}\left(x_{f}, x, t_{f}, t\right)$ be a generating function for the given phase flow. Then, $F_{1}$ satisfies the necessary conditions of the TPBVP by definition. Also, the function

$$
V(x, t)=-F_{1}\left(x_{f}, x, t_{f}, t\right)+\phi\left(x_{f}, t_{f}\right) \text { on } \psi\left(x_{f}, t_{f}\right)=0
$$

is the optimal cost function and satisfies the HJBE and the sufficient conditions. Furthermore, the optimal control law can be expressed as

$$
u=\arg \min _{\bar{u}} \bar{H}\left(x, \frac{\partial V(x, t)}{\partial x}, \bar{u}, t\right)
$$

Proof Refer to Park and Scheeres ${ }^{1,19}$.

In our previous works ${ }^{1,2,20}$, we have used generating functions and their Legendre transformations to develop a systematic methodology to solve a class of optimal control problems where the performance index and the system are analytic, and thus expandable as Taylor series. However, though we obtain a consistent result for problems with control constraints and singular optimal control problems, ${ }^{14}$ the applicability of our solution techniques is restrained by the unknown switching structure a priori, non-smoothness of cost function and control scheme, etc. In an effort to overcome these difficulties, we derive a new set of equations from the HJE and employ their solution to obtain the optimal control strategy, which we detail in the next section.

\section{Optimal Control Strategy Derived from the Hamiltonian Cauchy Problem}

\section{Derivation of Governing Equations}

We start from the HJEs for generating functions. First consider the HJE for $F_{1}$ in (9) and (11). Regarding $x_{0}$ and $t_{0}$ as constants (which is consistent with the definition of $F_{1}$ ) and taking partial differentiation of (11) with respect to $x$, we have

$$
\frac{\partial}{\partial x}\left(\frac{\partial F_{1}}{\partial t}+H\right)=0
$$

Here note that the Hamiltonian $H(x, \lambda, t)=H\left(x, \lambda\left(x, x_{0}, t, t_{0}\right), t\right)$ from (9). Using the chain rule for the Hamiltonian and the exactness property of $F_{1}$ yields

$$
\frac{\partial^{2} F_{1}}{\partial t \partial x}+\frac{\partial H}{\partial x}+\frac{\partial H}{\partial \lambda} \frac{\partial \lambda}{\partial x}=0
$$

Substituting $\lambda=\partial F_{1} / \partial x$ into the first term, we obtain a system of PDEs for the costate $\lambda$ :

$$
\frac{\partial \lambda}{\partial t}+\frac{\partial \lambda}{\partial x} \frac{\partial H}{\partial \lambda}=-\frac{\partial H}{\partial x}
$$

which is our new governing equation for the Hamiltonian system. Also starting from the HJE for $F_{2}$ in (12) and (14), taking $\lambda_{0}$ and $t_{0}$ as constants, and following the similar procedure, yields the same result. 
Now we derive a similar equation for the state $x$ from the HJEs for $F_{3}$ and $F_{4}$. From the HJE for $F_{3}$ in (15) and (17), if we regard $x_{0}$ and $t_{0}$ as constants and take partial derivatives of (17) with respect to $\lambda$, we have

$$
\frac{\partial}{\partial \lambda}\left(\frac{\partial F_{3}}{\partial t}+H\right)=0
$$

Observing that the $H(x, \lambda, t)=H\left(x\left(\lambda, x_{0}, t, t_{0}\right), \lambda, t\right)$ from (15) and using the chain rule for the Hamiltonian and the exactness property of $F_{3}$ yields

$$
\frac{\partial^{2} F_{3}}{\partial t \partial \lambda}+\frac{\partial H}{\partial \lambda}+\frac{\partial H}{\partial x} \frac{\partial x}{\partial \lambda}=0
$$

Substituting $x=-\partial F_{3} / \partial \lambda$ into the first term, we obtain a system of PDEs for the state $x$ :

$$
\frac{\partial x}{\partial t}-\frac{\partial x}{\partial \lambda} \frac{\partial H}{\partial x}=\frac{\partial H}{\partial \lambda},
$$

which is another set of governing equations for the Hamiltonian system. Finally starting from the HJE for $F_{4}$ in (18) and (20) and following a similar procedure yields the same result.

Note that (24) and (25) are $n$ simultaneous first order quasilinear PDEs. In order to solve these equations, we need to derive at least $n$ initial or terminal conditions to form an initial or terminal value problem. We see that the states $x$ and costates $\lambda$ in (24) and (25) are simply the same quantities as those in the Hamiltonian formulation in (3)-(5). Hence the boundary conditions for (24) and (25) should be compatible with those of the Hamiltonian system, that is, (7) and (8). We note that it is the type of boundary conditions (7) and (8) that determines which equations to use between (24) and (25), and how to use them to derive the optimal control scheme.

First we consider the hard constraint boundary condition (7). As this should be satisfied by the state $x$ in (25) at the terminal time, we obtain

$$
x\left(t=t_{f}, \lambda\right)=x_{f}
$$

Similarly for the soft constraint boundary condition (8), as this should be satisfied by (24) at the terminal time, we have

$$
\lambda\left(t=t_{f}, x\right)=\frac{\left.\partial\left[\phi\left(x, t_{f}\right)+\nu^{T} \psi(x), t\right)\right]}{\partial x}
$$

With these terminal conditions (26) and (27), the governing PDEs (25) and (24), respectively, constitute the Cauchy problems ${ }^{\mathrm{a}}$.

Finally note that the solutions to these Cauchy problems $(24,27)$ and $(25,26)$ are subordinate to generating functions by the relations (9), (12), (15) and (18); we can obtain the solutions to the above Cauchy problems simply by partial differentiations of generating functions, once we find them. However, as the motivation of this study suggests, it is very difficult to solve the HJE for a generating function for problems with control bounds, and thus non-smooth optimal control logic and cost function. In that case, we can resort to our new tools (24) and (25).

\section{Generation of Optimal Control Strategy}

So far we have derived a new set of governing equations and their associated terminal conditions to form a Cauchy problem. It remains how to evaluate the optimal control logic from these new formulations. We discuss the hard and soft constraint problem separately.

Suppose we have solved the Cauchy problem $(25,26)$ for the hard constraint problem. Then we have a solution of the form $x=x(t, \lambda)$. Again note that the state $x$ is the same variable as that of the Hamiltonian formulation (3)-(5); the

${ }^{a}$ Whereas our derivation originates from the Hamiltonian system theory, one can also derive the same results from the so-called invariant imbedding method based on characteristic theory of 1 st order PDEs. See Meyer ${ }^{21}$ for details. 
initial conditions $\left(x_{0}, \lambda_{0}\right)$ at the arbitrary moment $t_{0} \leq t_{f}$ should be satisfied by the solution to the Cauchy problem. Then given the (arbitrary) initial state $x_{0}$, the following equation should be satisfied:

$$
x\left(t_{0}, \lambda_{0}\right)=x_{0},
$$

which is an $n$-tuple of implicit algebraic equation for the $n$-tuple of unknowns $\lambda_{0}=\left[\begin{array}{llll}\lambda_{10} & \lambda_{20} & \cdots & \lambda_{n 0}\end{array}\right]^{T}$. If we find a solution $\lambda_{0}$ to this equation, we can evaluate the optimal trajectory by simple forward integration of (4)-(5). Furthermore solving (28) implicitly for a given domain of initial state to construct $\lambda_{0}=\lambda_{0}\left(t_{0}, x_{0}\right)$, we obtain the optimal feedback scheme by the optimality condition (6):

$$
u^{*}(x, \lambda(t, x), t)=\arg \min _{\bar{u}} \bar{H}(x, \lambda(t, x), \bar{u}, t)
$$

Note that we do not solve the Cauchy problem $(25,26)$ repetitively. Once we find a solution field for the domain of interest, the optimal feedback scheme can be obtained algebraically, which provides a substantial advantage over repetitive solving the TPBVP numerically for each boundary conditions.

For the soft constraint problem, the situation is more favorable, as is seen below. Similarly we first solve the Cauchy problem (24) and (27), which yields the solution of the form $\lambda=\lambda(t, x)$. Then the same arguments conclude that the following equation should be satisfied:

$$
\lambda\left(t_{0}, x_{0}\right)=\lambda_{0},
$$

Here note the difference from the hard constraint problem; given the initial state $x_{0}, \lambda_{0}$ is an explicit function of $t_{0}$ and $x_{0}$, which can be more easily computed in general. Then in the same way, starting from $\left(x_{0}, \lambda_{0}\right)$, we can evaluate the optimal trajectory as well as the optimal strategy.

Finally we conclude this section by claiming that our method is truly applicable to free final (or initial) time problems. In this case, the transversality condition for the free time index [15, section 2]

$$
\begin{aligned}
& H\left(t_{0}\right)-\frac{\partial \phi\left(x\left(t_{0}\right), t_{0}\right)}{\partial t_{0}}=0 \\
& H\left(t_{f}\right)+\frac{\partial \phi\left(x\left(t_{f}\right), t_{f}\right)}{\partial t_{f}}=0
\end{aligned}
$$

provides the additional algebraic equation for the varying time index.

\section{Numerical Computation}

So far we have shown that our new method is composed of two steps; first we solve the Cauchy problem $(25,26)$ or $(24,27)$, and then solve the associated implicit or explicit algebraic equations (28) or (30) for the hard or soft constraint problem to derive the optimal control scheme in feedback sense.

Though the well-posed Cauchy problem is guaranteed to have a unique solution [21, pages 9-17], it is by no means easy to solve a system of 1 st order quasilinear PDEs numerically for most non-trivial problems. Suppose we consider one of the traditional finite difference methods, for example. Then, we are first faced with the obstacle of dimensionality. If we assign $M$ grids for one spatial dimension and $N$ grids for the time span of interest, then we need $N M^{n}$ storage points for a $2 n$-dimensional Hamiltonian system representing the necessary conditions for optimality (3)-(5).

For the hard constraint problem, this curse of dimensionality becomes even more significant, as we need to solve the algebraic equations (30) implicitly. In general, we do not know a priori where in the $\lambda$-domain the solutions exist for the corresponding $x$-domain of interest. Thus, we need to solve the Cauchy problem $(25,26)$ for a large enough domain, in the hope that the solution falls into the estimated domain. For the soft constraint problem, the problem of estimating the solution domain can be alleviated, as the expression (30) becomes an explicit function for $x$. The initial costate $\lambda_{0}$ can be evaluated by $n$-dimensional interpolation, which is much simpler than the case of hard constraint problem. 
Despite these difficulties and limitations, note that once the Cauchy problems are solved, the solutions work as implicit (or explicit) numerical feedback charts for the hard (soft) constraint problem. We believe that with such charts the interpolation process can be done rapidly for relatively high dimensional problems. In other words, the solution to the Cauchy problem, the feedback chart, can be real time implementable for many practical problems.

\section{Illustrative Examples}

\section{Time Optimal Control of the Double Integrator System}

Consider minimizing

$$
J=\int_{t_{0}}^{t_{f}} d t
$$

subject to the double-integrator system with control constraints:

$$
\left[\begin{array}{c}
\dot{x}_{1} \\
\dot{x}_{2}
\end{array}\right]=\left[\begin{array}{c}
x_{2} \\
u
\end{array}\right],|u| \leq 1
$$

The initial and terminal boundary conditions are given by

$$
\left[\begin{array}{l}
x_{1}\left(t_{0}\right) \\
x_{2}\left(t_{0}\right)
\end{array}\right]=\left[\begin{array}{l}
x_{10} \\
x_{20}
\end{array}\right],\left[\begin{array}{l}
x_{1}\left(t_{f}\right) \\
x_{2}\left(t_{f}\right)
\end{array}\right]=\left[\begin{array}{l}
0 \\
0
\end{array}\right]
$$

Here for convenience, we fix the terminal time $t_{f}=0$ and vary the initial time $t_{0}$, which does not change the intrinsic property of the problem. Then, defining the pre-Hamiltonian as

$$
\bar{H}(x, \lambda, u, t)=1+\lambda_{1} x_{2}+\lambda_{2} u
$$

and using the Pontryagin's principle yields the following necessary conditions for optimality with the transversality condition for free initial time:

$$
\begin{aligned}
H & =1+\lambda_{1} x_{2}-\left|\lambda_{2}\right| \\
\dot{x}_{1} & =x_{2} \quad x_{1}\left(t_{0}\right)=x_{10} \quad x_{1}\left(t_{f}\right)=0 \\
\dot{x}_{2} & =-\operatorname{sign}\left(\lambda_{2}\right) \quad x_{2}\left(t_{0}\right)=x_{20} \quad x_{2}\left(t_{f}\right)=0 \\
\dot{\lambda}_{1} & =0 \\
\dot{\lambda}_{2} & =-\lambda_{1} \\
u & =-\operatorname{sign}\left(\lambda_{2}\right) \\
H\left(t_{0}\right) & =1+\lambda_{1}\left(t_{0}\right) x_{2}\left(t_{0}\right)-\left|\lambda_{2}\left(t_{0}\right)\right|=0
\end{aligned}
$$

From the transversality condition, we can show that there does not exist singular intervals and that the optimal control should be $u= \pm 1^{14}$. The Cauchy problem $(25,26)$ for this hard constraint problem can be written as

$$
\left[\begin{array}{c}
\frac{\partial x_{1}}{\partial t} \\
\frac{\partial x_{2}}{\partial t}
\end{array}\right]+\left[\begin{array}{ll}
\frac{\partial x_{1}}{\partial \lambda_{1}} & \frac{\partial x_{1}}{\partial \lambda_{2}} \\
\frac{\partial x_{2}}{\partial \lambda_{1}} & \frac{\partial x_{2}}{\partial \lambda_{2}}
\end{array}\right]\left[\begin{array}{c}
0 \\
-\lambda_{1}
\end{array}\right]=\left[\begin{array}{c}
x_{2} \\
-\operatorname{sign}\left(\lambda_{2}\right)
\end{array}\right],\left[\begin{array}{l}
x_{1}\left(t_{f}, \lambda\right) \\
x_{2}\left(t_{f}, \lambda\right)
\end{array}\right]=\left[\begin{array}{l}
0 \\
0
\end{array}\right]
$$

which can be solved numerically. In fact, this problem can be used to show that the solution to the above Cauchy problem can be derived from the associated generating functions, which has been computed in our previous work ${ }^{14}$. For example, starting from $F_{2}$ generating function, we can obtain the initial state $x_{0}$ as a function of terminal state $x_{f}$ and initial costate $\lambda_{0}$ :

$$
F_{2}\left(x_{f}, \lambda_{0}, t\right)=\frac{ \pm \lambda_{20}^{2}-2 \lambda_{20} \mp 1}{2 \lambda_{10}} \mp x_{2 f}+x_{1 f} \lambda_{10} \mp \frac{x_{2 f}^{2} \lambda_{10}}{2}, \quad(u=\mp 1 \rightarrow \pm 1)
$$

\footnotetext{
${ }^{\mathrm{b}}$ For the effective domain for each case of the solution, see also the same work. ${ }^{14}$
} 


$$
\begin{aligned}
x_{10} & =\frac{\partial F_{2}}{\partial \lambda_{10}}=x_{1 f} \mp \frac{1}{2} x_{2 f}^{2}+\frac{\mp \lambda_{20}^{2}+2 \lambda_{20} \pm 1}{2 \lambda_{10}^{2}} \\
x_{20} & =\frac{\partial F_{2}}{\partial \lambda_{20}}=\frac{ \pm \lambda_{20}-1}{\lambda_{10}}
\end{aligned}
$$

Imposing the given terminal boundary condition at the origin, i.e., $\left(x_{1 f}, x_{2 f}\right)=(0,0)$, and removing the subscript 0 to represent the moving initial conditions, we have

$$
\left(x_{1}, x_{2}\right)=\left(\frac{\mp \lambda_{2}^{2}+2 \lambda_{2} \pm 1}{2 \lambda_{1}^{2}}, \frac{ \pm \lambda_{2}-1}{\lambda_{1}}\right)
$$

Simply by direct substitution, we can easily show that this expression satisfies the above Cauchy problem.

Now suppose that we have found this solution numerically from the Cauchy problem $(25,26)$. Then fixing the initial states into the desired ones $\left(x_{1}, x_{2}\right)=\left(x_{10}, x_{20}\right)$, we can find the loci of initial costates from each component of the solution in the $\lambda_{1} \lambda_{2}$-domain. The whole procedure can be shown graphically with ease. We first draw plots for $x_{1}=x_{1}\left(\lambda_{1}, \lambda_{2}\right)$ and $x_{2}=x_{2}\left(\lambda_{1}, \lambda_{2}\right)$ (Figure 1). Then for the desired initial states, we construct the contours for each plot, as in Figure 1 where the contours are drawn for $x_{1}=0 \sim 1$ and $x_{2}=1 \sim 2$, respectively. Imposing these two contours together in the $\lambda_{1} \lambda_{2}$-plane, we can find the initial costates for the given initial states by choosing the intersection of these two contours (Figure 2). Then, the optimal control law can be determined from the optimality condition $u=-\operatorname{sign}\left(\lambda_{2}\right)$.
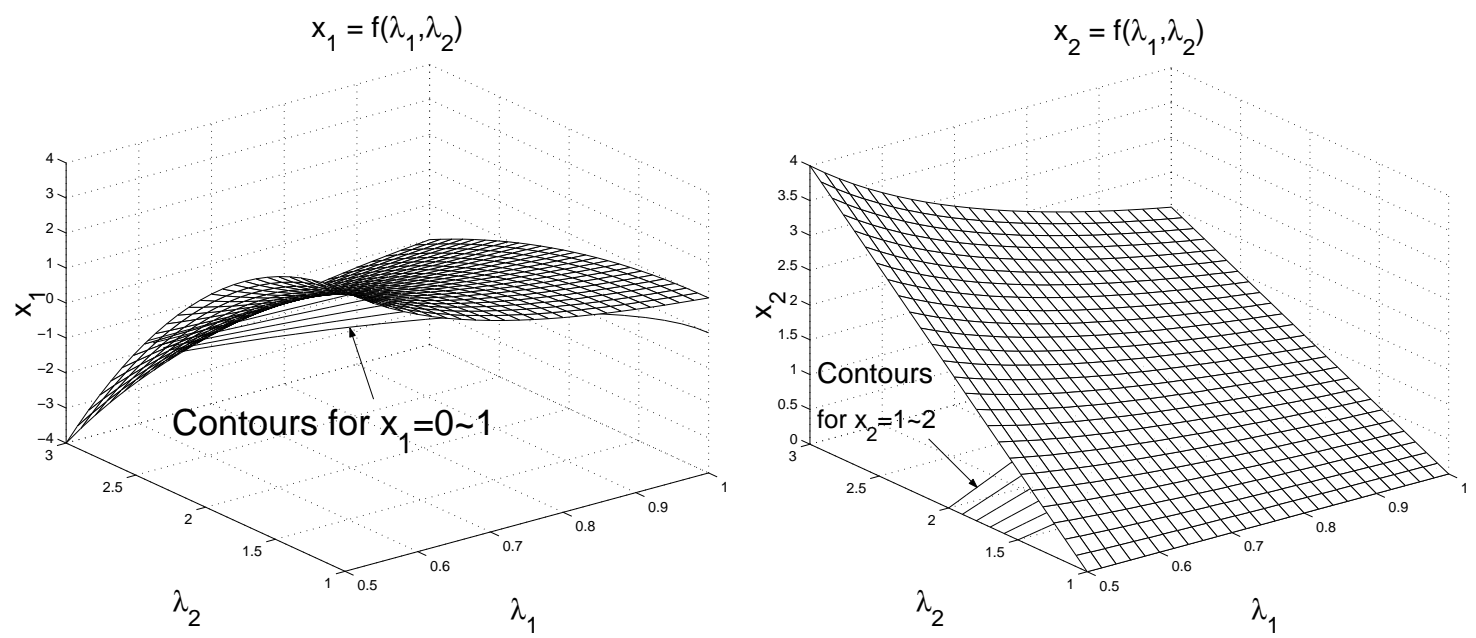

Figure 1. Solutions to the Cauchy Problem $\left(x_{f}=0\right)$

\section{Time-Optimal Control of a Harmonic Oscillator}

Consider minimizing

$$
J=\int_{t_{0}}^{t_{f}} d t
$$

subject to the second order system with control constraints

$$
\left[\begin{array}{c}
\dot{x}_{1} \\
\dot{x}_{2}
\end{array}\right]=\left[\begin{array}{c}
x_{2} \\
-\epsilon x_{1}+a u
\end{array}\right],|u| \leq 1
$$




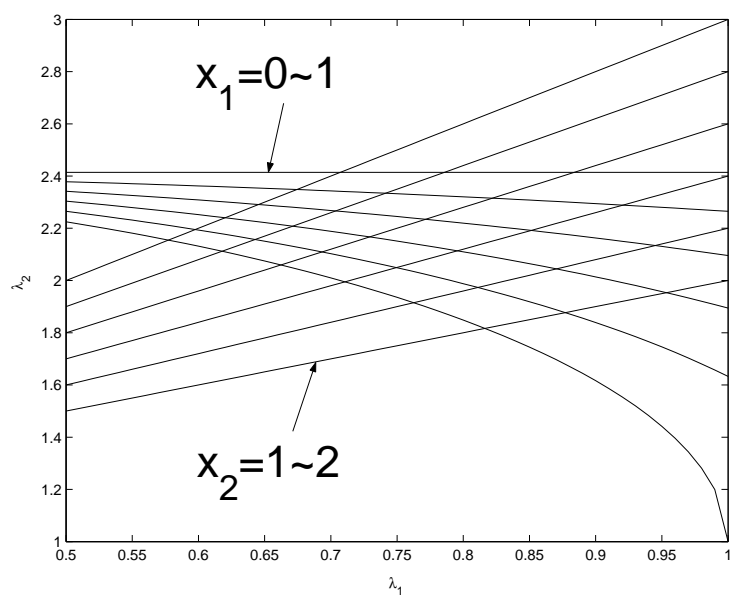

Figure 2. Graphical Determination of Initial Costate $\left(x_{f}=0\right)$. Each intersection point defines a unique pair of $\left(\lambda_{1}, \lambda_{2}\right)$ at $\left(x_{1}, x_{2}\right)$.

satisfying the initial and terminal boundary conditions

$$
\left[\begin{array}{l}
x_{1}(0) \\
x_{2}(0)
\end{array}\right]=\left[\begin{array}{l}
x_{10} \\
x_{20}
\end{array}\right], \quad\left[\begin{array}{l}
x_{1}\left(t_{f}\right) \\
x_{2}\left(t_{f}\right)
\end{array}\right]=\left[\begin{array}{l}
0 \\
0
\end{array}\right] .
$$

Here $a>0$ and $\epsilon \geq 0$ are constant parameters. Defining the pre-Hamiltonian $\bar{H}$ as

$$
\bar{H}=1+\lambda_{1} x_{2}+\lambda_{2}\left(-\epsilon x_{1}+a u\right)
$$

and referring to the Pontryagin's principle, we obtain the necessary conditions for optimality with the transversality condition for free initial time:

$$
\begin{aligned}
H & =1+\lambda_{1} x_{2}-\epsilon \lambda_{2} x_{1}-a\left|\lambda_{2}\right| \\
\dot{x}_{1} & =x_{2} \\
\dot{x}_{2} & =-\epsilon x_{1}-a \operatorname{sign}\left(\lambda_{2}\right) \\
\dot{\lambda}_{1} & =\lambda_{2} \\
\dot{\lambda}_{2} & =-\lambda_{1} \\
u & =-\operatorname{sign}\left(\lambda_{2}\right) \\
\left.H(x, \lambda, t)\right|_{t=t_{0}} & =1+\lambda_{1}\left(t_{0}\right) x_{2}\left(t_{0}\right)-\epsilon \lambda_{2}\left(t_{0}\right) x_{1}\left(t_{0}\right)-\left|\lambda_{2}\left(t_{0}\right)\right|=0,
\end{aligned}
$$

It can be shown from the transversality condition that there does not exist any singular intervals. In order to further examine the switching structure, note that the costate dynamics represent the unforced harmonic oscillator; letting $\epsilon=\omega^{2}$, we obtain

$$
\left[\begin{array}{l}
\lambda_{1}(t) \\
\lambda_{2}(t)
\end{array}\right]=\left[\begin{array}{cc}
\cos \omega t & \sin \omega t \\
-\sin \omega t & \cos \omega t
\end{array}\right]\left[\begin{array}{c}
\lambda_{10} \\
\lambda_{20}
\end{array}\right]
$$

Considered with the control logic (33), this shows that unlike the double integrator problem there can be multiple discontinuous switchings in the control flow depending on the initial conditions.

With this structure the Cauchy problem $(25,26)$ is formulated as

$$
\left[\begin{array}{c}
\frac{\partial x_{1}}{\partial t} \\
\frac{\partial x_{2}}{\partial t}
\end{array}\right]+\left[\begin{array}{cc}
\frac{\partial x_{1}}{\partial \lambda_{1}} & \frac{\partial x_{1}}{\partial \lambda_{2}} \\
\frac{\partial x_{2}}{\partial \lambda_{1}} & \frac{\partial x_{2}}{\partial \lambda_{2}}
\end{array}\right]\left[\begin{array}{c}
\epsilon \lambda_{2} \\
-\lambda_{1}
\end{array}\right]=\left[\begin{array}{c}
x_{2} \\
-\epsilon x_{1}-a \operatorname{sign}\left(\lambda_{2}\right)
\end{array}\right],\left[\begin{array}{l}
x_{1}\left(t_{f}, \lambda\right) \\
x_{2}\left(t_{f}, \lambda\right)
\end{array}\right]=\left[\begin{array}{l}
0 \\
0
\end{array}\right] .
$$


Before proceeding further, observe from the non-smooth term $\operatorname{sign}\left(\lambda_{2}\right)$ that the solution to this Cauchy problem is expected to develop shocks around the switching surface. Properly interpreting this phenomenon, we should be cautious in selecting appropriate numerical methods. As far as the finite difference method is concerned, it turns out by numerical experiment that the 1st order upwind scheme is capable of analyzing the shocks properlyc; unlike the latter, the former switches between forward and backward differences in spatial dimensions by reflecting the characteristic directions of the associated PDE, which are transmitted by the sign of $\lambda_{1}$ and $\lambda_{2}$ at each grid.

As particular examples, a harmonic oscillator $(\epsilon=1, a=1)$ is compared with a double integrator $(\epsilon=0, a=1)$, which can be also viewed as an extreme case of a harmonic oscillator. Then as suggested to properly interpret the shocks, we launch the 1st order upwind scheme for temporal and spatial domains $0 \leq t \leq 1$ and $-4 \leq \lambda_{1}, \lambda_{2} \leq 4$ with the relevant grids $\Delta t=0.02$ and $\Delta \lambda_{1}=\Delta \lambda_{2}=0.04$ respectively. As this yields a numerical solution in the form of $\left(x_{1}, x_{2}\right)=f\left(t, \lambda_{1}, \lambda_{2}\right)$, it provides numerical charts with which to compute the initial costate for the desired initial state by interpolation with the implicit relation (28). Then the optimal control law is simply obtained from (29). Recall that this process has been graphically illustrated in the previous example. For comparison purpose, we portray collections of initial states with the same optimal time for each case, which can be achieved by forcing the transversality conditions (34) at each time step. Figure 3 clearly displays the distinct patterns of these collections, together with the different shapes of switching curves between each example.

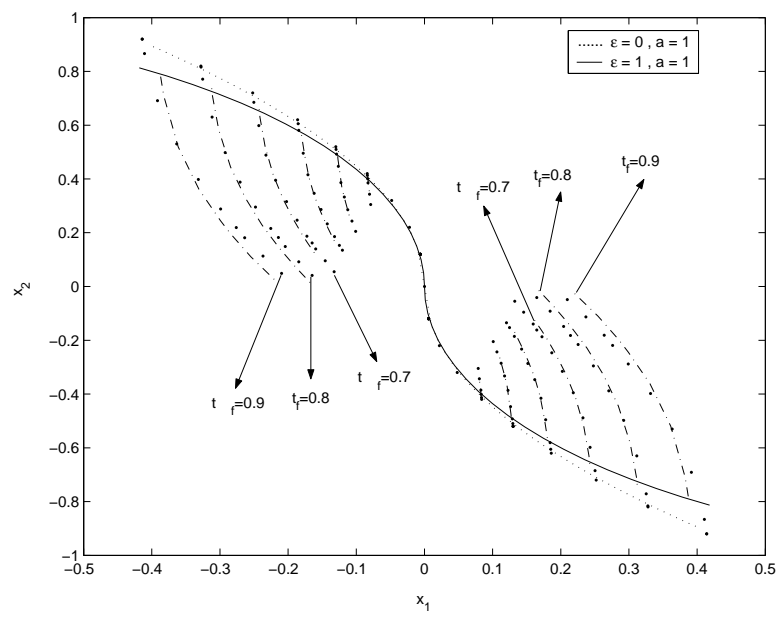

Figure 3. Collection of the Initial States with the Same Optimal Time and Switching Curves for $(\epsilon=1, a=1)$ and $(\epsilon=0, a=1)$

\section{Optimal Control of a Spacecraft Orbital Maneuvers}

As a main application, we now study optimal control of spacecraft orbital maneuvers. Specifically we deal with fuel-optimal maneuvers of a spacecraft in a central gravity field: minimize

$$
J=\frac{1}{2} x^{T}\left(t_{f}\right) Q_{f} x\left(t_{f}\right)+T \int_{t_{0}}^{t_{f}}|u(t)| d t
$$

subject to the system

$$
\begin{aligned}
\dot{x} & =v \\
\dot{v} & =A x+B v+u
\end{aligned}
$$

${ }^{c}$ Refer to Appendix for a brief description of this method. For a general discussion on finite difference methods, we cite Thomas ${ }^{22}$ 
Here $x=\left[x_{1} x_{2}\right]^{T}, v=\left[\begin{array}{ll}v_{1} & v_{2}\end{array}\right]^{T}, u=\left[\begin{array}{ll}u_{1} & u_{2}\end{array}\right]^{T}$, and $t_{f}$ is assumed to be fixed. $A$ and $B$ are defined respectively as

$$
A=\left[\begin{array}{ll}
3 & 0 \\
0 & 0
\end{array}\right] \quad, \quad B=\left[\begin{array}{cc}
0 & 2 \\
-2 & 0
\end{array}\right]
$$

This system is called the Clohessy-Wiltshire equation and represents the linearized planar motion of a particle in a inverse-square gravity field, with its coordinates expressed in a circular rotating coordinate frame. For more detailed description of this system, we cite Carter. ${ }^{8}$

Defining the pre-Hamiltonian as

$$
\bar{H}=|u|+p^{T} v+q^{T}(A x+B v+b u)
$$

we can obtain the costate equations

$$
\begin{aligned}
\dot{p} & =-A^{T} q \\
\dot{q} & =-p-B^{T} q
\end{aligned}
$$

and the optimal control logic by Pontryagin's principle:

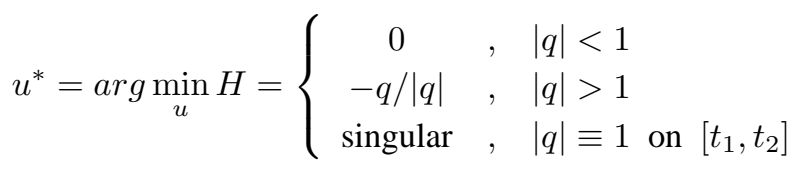

According to Carter, ${ }^{8}$ the singular arc occurs only when $q=[ \pm 10]^{T}$. For the sake of simplicity, we do not consider this special case. Then introducing the regular optimal control logic into the pre-Hamiltonian $\bar{H}$ yields the Hamiltonian as a function of states $(r, v)$ and costates $(p, q)$ only.

\section{Hard Constraint Problem}

We first consider rendezvous-type boundary conditions:

$$
\begin{array}{ll}
x\left(t_{0}\right)=x_{0} & , \quad x\left(t_{f}\right)=x_{f} \\
v\left(t_{0}\right)=v_{0} & , \quad v\left(t_{f}\right)=v_{f}
\end{array}
$$

Without loss of generality, we can set $Q_{f}=0$. Then similarly as in the previous example, we can construct the Cauchy problem for this hard constraint problem:

$$
\frac{\partial \bar{x}}{\partial t}=A_{1} \frac{\partial \bar{x}}{\partial \lambda_{1}}+A_{2} \frac{\partial \bar{x}}{\partial \lambda_{2}}+A_{3} \frac{\partial \bar{x}}{\partial \lambda_{3}}+A_{4} \frac{\partial \bar{x}}{\partial \lambda_{4}}+C_{0} \bar{x}+c_{1}, \bar{x}\left(t_{f}, \bar{\lambda}\right)=\left[x\left(t_{f}, \bar{\lambda}\right)^{T} v\left(t_{f}, \bar{\lambda}\right)^{T}\right]^{T}
$$

where

$$
\begin{aligned}
& \bar{x}=\left[\begin{array}{l}
x \\
v
\end{array}\right], \bar{\lambda}=\left[\begin{array}{l}
p \\
q
\end{array}\right], C_{0}=\left[\begin{array}{cc}
0 & I \\
A & B
\end{array}\right], C_{1}=\left[\begin{array}{l}
0 \\
u
\end{array}\right] \\
& A_{1}=3 \lambda_{3}, A_{2}=0, A_{3}=\lambda_{1}-\lambda_{4}, A_{4}=\lambda_{2}+\lambda_{3} .
\end{aligned}
$$

We now launch the 1st order upwind finite difference scheme to properly interpret the non-smoothness of control $\operatorname{logic}^{\mathrm{d}}$. As a specific example, we locate the terminal boundary condition at the origin, and consider time span $[0,0.1]$. Temporal grid and spatial grids have been chosen as $\Delta t=0.01$ and $\Delta \lambda_{i}=0.02, i=1 \sim 4$ respectively. As this yields a numerical solution in the form of $\bar{x}=f(t, \bar{\lambda})$, it provides numerical charts with which to compute the initial costate

\footnotetext{
${ }^{\mathrm{d}}$ Again refer to Appendix for a brief description of this method, or to Thomas ${ }^{22}$ for a general discussion on finite difference methods.
} 
for the desired initial state by the implicit relation (28). Then the optimal strategy is obtained from the Pontryagin's principle (29). All these numerical experiments have been performed with Matlab ${ }^{\circledR}$. It should be emphasized that we do not need to guess the initial costate in contrast to the direct numerical solution to the two point boundary value problem (TPBVP) for the Hamiltonian system (4)-(5). Also we do not need to solve the Cauchy problem again as long as our desired initial states implicitly remain in the numerical solution chart.

To test the validity of our method, we have chosen some arbitrary initial states around the origin, obtain the initial costates for them by (29), and solve the initial value problem for the Hamiltonian system (4)-(5). Figure 4 shows the state trajectories in the position and velocity phase plane for those initial conditions. Here the slight offset of the terminal states from the origin is mainly caused by relatively coarse grids chosen above. As $\Delta t$ and $\Delta \lambda_{i}$ become smaller, the terminal states come closer to the origin. Figure 5 shows the optimal control schemes for the given initial conditions. It is apparent that our method captures non-smooth control profiles, as well as smooth ones.
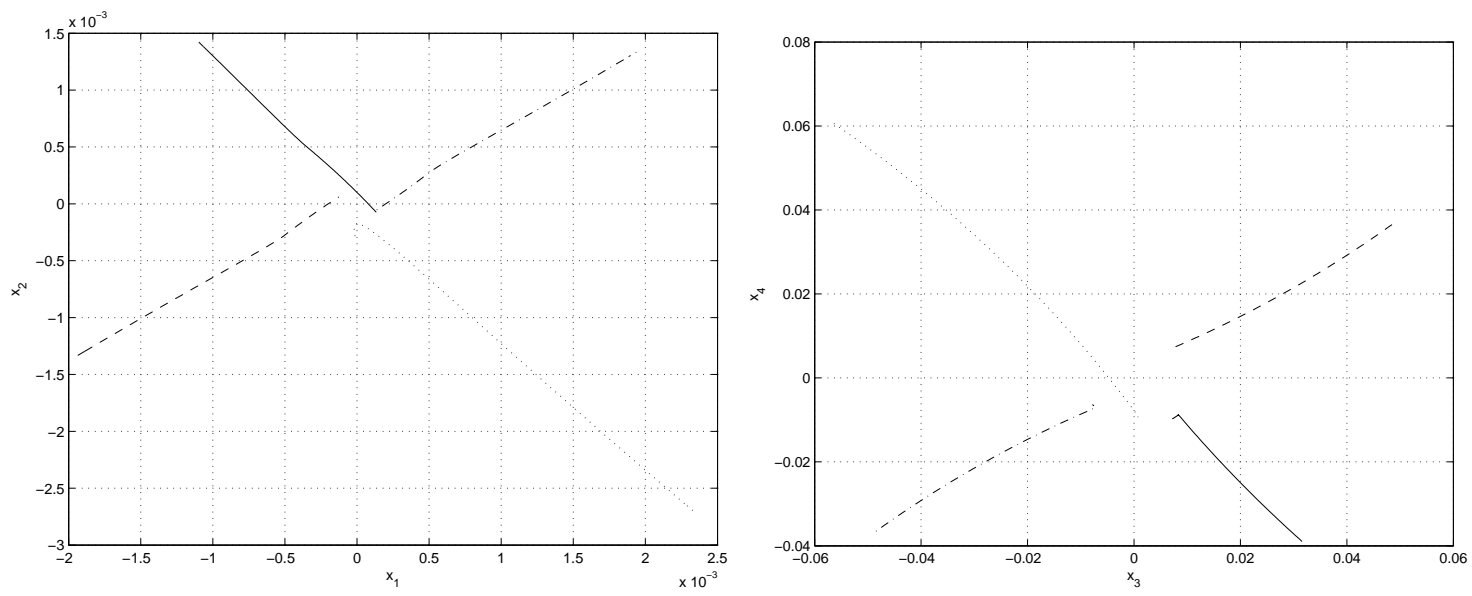

Figure 4. State Trajectory in the Position and Velocity Phase Plane $\left(\bar{x}_{f}=0\right)$
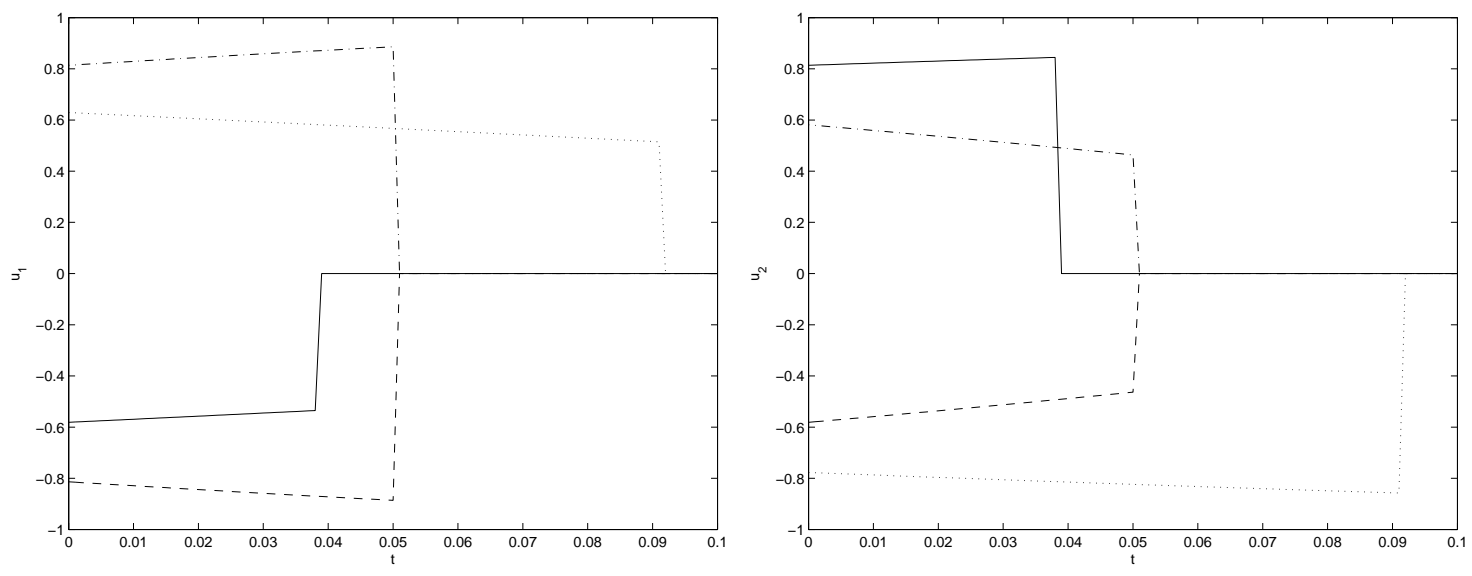

Figure 5. Time History of Control Variable $\left(\bar{x}_{f}=0\right)$

Throughout this method, the main concern lies in the data storage capacity, as this formulation deals with 1 temporal and 4 spatial variables. For example, working with 100 temporal grids and 100 spatial grids in each dimension, we need $4 \times 10^{10}$ memory allocations for the Cauchy problem solution in the form of $\bar{x}=f(t, \bar{\lambda})$, which is formidable even with modern standard computing facilities. With this 'curse' of dimensionality, it is difficult to compute for a large domain with relatively small grids providing tolerable accuracy. This is reflected by rather apparent offsets of 
terminal states from the origin in Figure 4. Even in order to evaluate those trajectories for those initial conditions in Figure 4-5, we have first divided the $\lambda$-domains of our interest into a few sub-domains to reduce grid sizes to an acceptable degree as well as not to exceed memory allocation capacity, and applied the finite difference scheme separately to each of those sub-domains.

Another concern, which is only confined to the hard constraint formulation, lies that the relation (28) yielding the initial costate is implicit. Recall that we desire to obtain its inverse relation to derive the optimal strategy from the Pontragin's principle (29) in feedback sense; it is not easily pliable to construct an inverse mapping for 5-dimensional arrays. This is mainly responsible for demonstrating the open-loop trajectory only by solving the initial value problem of the relevant Hamiltonian system. In fact, this difficulty of obtaining the inverse relation for $\bar{\lambda}$ is more relevant to moderately acceptable achievement of terminal constraint in Figure 4. Once we obtain the relation $\bar{\lambda}=f(t, \bar{x})$, we can easily update our control strategy as new measurements are made, which should provide higher accuracy with relatively coarse grids. This is explicitly demonstrated in a soft constraint formulation below.

\section{Soft Constraint Problem}

In order to study the soft constraint formulation, we first see that all the above derivation holds except that we now have a transversality condition for the terminal boundary condition:

$$
\bar{\lambda}\left(t_{f}\right)=Q_{f} \bar{x}\left(t_{f}\right)
$$

We can now apply the general Cauchy problem formula $(24,27)$ to this soft constraint problem:

$$
\frac{\partial \bar{\lambda}}{\partial t}=A_{1} \frac{\partial \bar{\lambda}}{\partial x_{1}}+A_{2} \frac{\partial \bar{\lambda}}{\partial x_{2}}+A_{3} \frac{\partial \bar{\lambda}}{\partial x_{3}}+A_{4} \frac{\partial \bar{\lambda}}{\partial x_{4}}+C_{0} \bar{\lambda}, \quad \bar{\lambda}\left(t_{f}, \bar{x}\right)=Q_{f} \bar{x}
$$

where

$$
\begin{aligned}
C_{0} & =\left[\begin{array}{cc}
0 & A_{s} \\
-I & B_{s}
\end{array}\right], A_{s}=\left[\begin{array}{cc}
-3 & 0 \\
0 & 0
\end{array}\right], B_{s}=\left[\begin{array}{cc}
0 & 1 \\
-1 & 0
\end{array}\right] \\
A_{1} & =-v_{1}, A_{2}=-v_{2}, A_{3}=-3 x_{1}-2 v_{2}-u_{1}(q), A_{4}=2 v_{1}-u_{2}(q) .
\end{aligned}
$$

Similarly as in the hard constraint problem, we execute the 1st order upwind finite difference scheme. As a specific example, we set $Q_{f}=5 I$ and choose the time span $[0,1]$. Temporal grids and spatial grids have been chosen as $\Delta t=0.1$ and $\Delta x_{i}=0.2, i=1 \sim 4$ respectively. As this yields a numerical solution in the form of $\bar{\lambda}=f(t, \bar{x})$, it provides numerical charts from which the initial costate can be obtained for the desired initial state by the explicit relation (30). Again all these numerical experiments have been performed with Matlab ${ }^{\circledR}$.

Figure 6 shows the state trajectory in the position and velocity phase plane for some arbitrarily chosen initial conditions to test the validity of our method. Here the solid lines represent trajectories obtained from the Cauchy problem approach. It should be emphasized that we have evaluated these trajectories in feedback sense; as the trajectory flows, we have reevaluated the optimal control scheme at every $\delta t=0.02$ interval using the numerical solution to the Cauchy problem. The dotted lines represent reference trajectories evaluated by solving the TPBVP using the forward shooting method numerically. For their initial guesses on initial costates, we have used the Cauchy problem solution at the initial time. Relatively big discrepancies between each solution on the velocity plane for some initial conditions are caused by chattering on the boundaries of full/null thrust.

Finally note that our numerical concerns regarding the curse of dimensionality can be mitigated for the soft constraint formulation. As the associated Cauchy problem $(24,27)$ directly provides the costate as a function of states, we can initially estimate the domain of numerical evaluation, in contrast to the case of hard constraint problem. Furthermore once we can implement feedback control scheme during the mission, we can accomplish more accurate trajectories than those obtained in open-loop sense with relatively coarse grids. This statement can be supported by comparing the hard/soft constraint formulations above together; it can be observed that we have obtained very close approximation to the reference solution with even higher grid sides and longer time span in the soft constraint formulation than in the hard constraint formulation. 

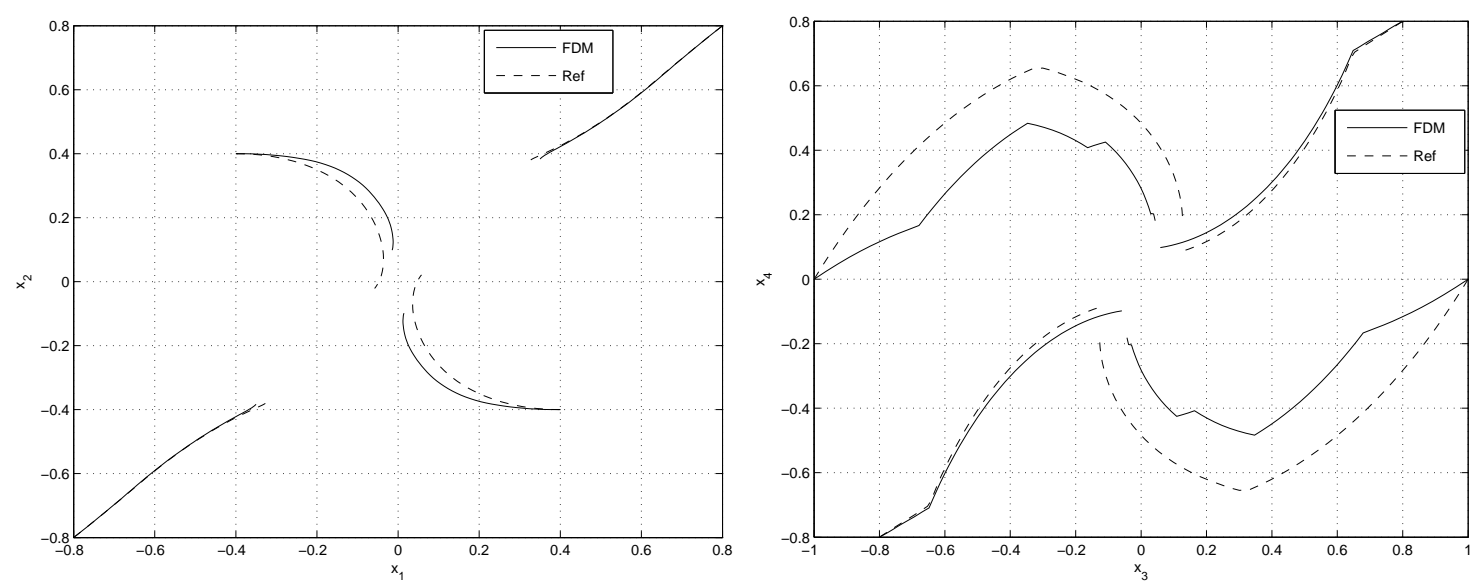

Figure 6. State Trajectory in the Position and Velocity Phase Plane $\left(Q_{f}=5 I\right)$

\section{Conclusion}

We have applied the Hamilton-Jacobi theory to present a new method for solving the optimal control problem in feedback sense. Formulating the optimal control problem as a Hamiltonian system, we have derived a set of partial differential equations with their associated boundary conditions to form Cauchy problems. Then it has been shown how the solutions to the Cauchy problems can be used for solving both hard and soft constraint problems. Finally our technique has been extensively used to study optimal control problems for spacecraft orbital maneuvers dominated by non-smooth control logic. For the hard constraint problem, our solution satisfies the given boundary conditions with tolerable offset, which is mainly caused by relatively coarse grids. For the soft constraint problem, we have executed the feedback control scheme to evaluate the optimal trajectories, which has been favorably compared with the reference trajectories, even with relatively coarse grids. With these encouraging results, we believe that our technique, along with other traditional techniques, can be considered as an alternative method for non-smooth optimal control problems.

In the future, we focus more on computational issues; more effective numerical scheme for solving Cauchy problems, more efficient storage for vast Cauchy problem data in high dimensional problems, enhancement of the implicit/explicit interpolation process, etc. These issues will accompany researches on more realistic nonlinear spacecraft orbital maneuver formulations.

\section{Appendix: Finite Difference Method for Solving Cauchy Problems}

As a concise reference, we briefly describe the 1st order upwind scheme, a finite difference method used to solve Cauchy problems numerically. For a comprehensive discussion on a variety of finite difference methods, we cite Thomas. ${ }^{22}$

Consider a general Cauchy problem of the form:

$$
\frac{\partial x(t, \lambda)}{\partial t}+\frac{\partial x(t, \lambda)}{\partial \lambda} G(x, \lambda, t)=F(x, \lambda, t), x\left(t=t_{0}, \lambda\right)=f(\lambda)
$$

If we assume that $t \in \mathbf{R}$ and $x, \lambda, G, F, f \in \mathbf{R}^{2}$, we can rearrange this equation as

$$
\frac{\partial x}{\partial t}=A_{1} \frac{\partial x}{\partial \lambda_{1}}+A_{2} \frac{\partial x}{\partial \lambda_{2}}+C_{0} x+c_{1}
$$

where $A_{1}, A_{2} \in \mathbf{R}, C_{0} \in \mathbf{R}^{2 \times 2}$, and $c_{1} \in \mathbf{R}^{2}$. Now denoting the temporal grid by $\Delta t$ and the spatial grids by $\Delta \lambda_{1}$ 
and $\Delta \lambda_{2}$ respectively, we can derive the 1st order upwind scheme as follows:

$$
\begin{array}{r}
x_{j k}^{n+1}=x_{j k}^{n}+R_{\lambda_{1}} A_{1} \delta x_{M k}^{n}+R_{\lambda_{2}} A_{2} \delta x_{j N}^{n}+\Delta t\left(C_{0} x_{j k}^{n}+c_{1}\right) \\
R_{\lambda_{1}}=\frac{\Delta t}{\Delta \lambda_{1}}, R_{\lambda_{2}}=\frac{\Delta t}{\Delta \lambda_{2}} \\
\delta x_{M k}^{n}=x_{M k}^{n}-x_{(M-1) k}^{n}, M=\left\{\begin{array}{lll}
j & \text { if } & A_{1}>0 \\
j+1 & \text { if } & A_{1}<0
\end{array}\right. \\
\delta x_{j N}^{n}=x_{j N}^{n}-x_{j(N-1)}^{n}, N=\left\{\begin{array}{lll}
k & \text { if } & A_{2}>0 \\
k+1 & \text { if } & A_{2}<0
\end{array}\right.
\end{array}
$$

Observe that this algorithm switches between forward and backward difference schemes in spatial dimensions depending on the signs of $A_{1}$ and $A_{2}$, which reflects the characteristic directions at each grid of evaluation. Here the superscript and subscript represent the temporal and spatial indices respectively.

\section{Acknowledgement}

This research is supported by National Science Foundation Grant CMS 0408542. We acknowledge fruitful discussions with Prof. Philip Roe concerning numerical solutions of Cauchy problems.

\section{References}

${ }^{1}$ Park, C. and Scheeres, D. J., "Solutions of Optimal Feedback Control Problem with General Boundary Conditions using Hamiltonian Dynamics and Generating Functions," Automatica, Vol. 42, No. 5, 2006, pp. 869-875.

${ }^{2}$ Park, C., Guibout, V., and Scheeres, D. J., "Solving Optimal Continuous Thrust Rendezvous Problems with Generating Functions," Journal of Guidance, Control, and Dynamics, Vol. 29, No. 2, 2006, pp. 321-331.

${ }^{3}$ Lawden, D. F., Optimal Trajectories for Space Navigation, Butterworths, London, England, 1963.

${ }^{4}$ Clohessy, W. H. and Wiltshire, R. S., "Terminal Guidance System for Satellite Rendezvous," Journal of the Aerospace Sciences, Vol. 27, No. 9, 1960, pp. 653-.

${ }^{5}$ Anthony, M. L. and Sasaki, F. T., "Rendezvous Problem for Nearly Circular Orbits," AIAA Journal, Vol. 3, No. 9, 1965, pp. $1066-1073$.

${ }^{6}$ Euler, E. A., "Optimal Low-Thrust Rendezvous Control," AIAA Journal, Vol. 7, No. 6, 1969, pp. 1140-1144.

${ }^{7}$ Marec, J., Optimal Space Trajectories, Elsevier, New-York, NY, 1979.

${ }^{8}$ Carter, T. E., "Fuel-Optimal Maneuvers of a Spacecraft Relative to a Point in Circular Orbit," Journal of Guidance, Control, and Dynamics, Vol. 7, No. 6, 1984, pp. 710-716.

${ }^{9}$ Redding, D. C., "Highly Efficient, Very Low Thrust Transfer to Geosynchronous Orbit: Exact and Approximate Solutions," Journal of Guidance, Control, and Dynamics, Vol. 7, No. 2, 1984, pp. 140-147.

${ }^{10}$ Carter, T. and Humi, M., "Fuel-Optimal Rendezvous Near a Point in General Keplerian Orbit," Journal of Guidance, Control, and Dynamics, Vol. 10, No. 6, 1989, pp. 567-573.

${ }^{11}$ Carter, T. E. and Pardis, C. J., "Optimal Power-Limited Rendezvous with Upper and Lower Bounds on Thrust," Journal of Guidance, Control, and Dynamics, Vol. 19, No. 5, 1996, pp. 1124-1133.

${ }^{12}$ Kechichian, J. A., "Minimum-time Constant Acceleration Orbit Transfer with First Order Oblateness Effect," Journal of Guidance, Control, and Dynamics, Vol. 23, No. 4, 2000, pp. 595-603.

${ }^{13}$ Herman, A. L. and Spencer, D. B., "Optimal Low-Thrust Earth-Orbit Transfers using Higher-Order Collocation Methods," Journal of Guidance, Control, and Dynamics, Vol. 25, No. 1, 2002, pp. 40-47.

${ }^{14}$ Park, C. and Scheeres, D. J., "Extended Applications of Generating Functions to Optimal Feedback Control Problems," Proceedings of the American Control Conference, 2005, pp. 852-857, Portland, OR.

${ }^{15}$ Bryson, A. E. and Ho, Y., Applied Optimal Control, Hemisphere Publishing Corp., London, England, 1975.

${ }^{16}$ Guibout, V. and Scheeres, D. J., "Solving Relative Two Point Boundary Value Problems: Applications to Spacecraft Formation Flight Transfers," Journal of Guidance, Control, and Dynamics, Vol. 27, No. 4, 2004, pp. 693-704.

${ }^{17}$ Greenwood, D. T., Classical Dynamics, Prentice-Hall, Inc., Englewood Cliffs, NJ, 1977.

${ }^{18}$ Goldstein, H., Classical Mechanics, Addision-Wesley, Reading, MA, 1965.

${ }^{19}$ Park, C., The Hamilton-Jacobi Theory for Solving Optimal Feedback Control Problems with General Boundary Conditions, Ph. D. Thesis, University of Michigan, Ann Arbor, MI, 2006. 
${ }^{20}$ Park, C. and Scheeres, D. J., "Solutions of Optimal Feedback Control Problem using Hamiltonian Dynamics and Generating Functions," IEEE conference on Decision and Control, 2003, pp. 1222-1227, Maui, Hawaii.

${ }^{21}$ Meyer, G. H., Initial Value Methods for Boundary Value Problems, Academic Press, New York, NY, 1973.

${ }^{22}$ Thomas, J. W., Numerical Partial Differential Equations: Finite Difference Methods, Springer Verlag, New York, NY, 1995. 